\title{
Region-specific involvement of actin rearrangement- related synaptic structure alterations in conditioned taste aversion memory
}

\author{
Ai-Ling Bi, Yue Wang, Bo-Qin Li, Qian-Qian Wang, Ling Ma, Hui Yu, Ling Zhao, and \\ Zhe-Yu Chen ${ }^{1}$
}

Department of Neurobiology, Shandong Provincial Key Laboratory of Mental Disorders, School of Medicine, Shandong University, Jinan, Shandong 250012, People's Republic of China

\begin{abstract}
Actin rearrangement plays an essential role in learning and memory; however, the spatial and temporal regulation of actin dynamics in different phases of associative memory has not been fully understood. Here, using the conditioned taste aversion (CTA) paradigm, we investigated the region-specific involvement of actin rearrangement-related synaptic structure alterations in different memory processes. We found that CTA training could induce increased postsynaptic density (PSD) length in insular cortex (IC), but not in basolateral amygdala (BLA) and prelimbic cortex (PrL) during short-term memory (STM) formation, whereas it led to increased PSD length and synapse density in both IC and PrL during longterm memory (LTM) formation. Inhibition of actin rearrangement in the IC, but not in the BLA and PrL, impaired memory acquisition. Furthermore, actin dynamics in the IC or PrL is necessary for memory consolidation. On the contrary, inhibition of actin dynamics in the IC, BLA, or PrL had no effect on CTA memory retrieval. Our results suggest temporal and regional-specific regulation of actin rearrangement-related synaptic structure in different phases of CTA memory.
\end{abstract}

[Supplemental material is available online at http://www.learnmem.org.]

Conditioned taste aversion (CTA) is a type of associative memory in which the subject learns to associate a taste with delayed malaise (Bures et al. 1988; Cui et al. 2005). CTA has a number of unique temporal properties that distinguish it from other forms of learning, which is formed by single-trial training and is a longlasting memory with little or no loss of memory months after conditioning (Houpt et al. 1996). These characteristics make CTA a useful model to study the different phases of memory, such as acquisition, consolidation, and retrieval. The neural circuitry of CTA includes the insular cortex (IC), amygdala, thalamus, parabrachial nucleus, and nucleus of the solitary tract (Norgren 1995). In addition, new evidence has indicated that the ventromedial prefrontal cortex (vmPFC) plays a role in the extinction of CTA memory (Akirav et al. 2006; Yu et al. 2009).

The structural remodeling of synapses and formation of new synaptic contacts is a possible mechanism underlying the late phase of long-term potentiation, a form of plasticity that is involved in learning and memory (Bliss and Collingridge 1993; Buchs and Muller 1996; Maletic-Savatic et al. 1999). Actin is the major structural component of synapse, which has been shown to play an important role in synaptic plasticity by maintaining characteristically and highly dynamic transformation between G-actin and F-actin through actin rearrangement (Fifkova and Morales 1992; Fukazawa et al. 2003; Zhou et al. 2004; Zito et al. 2004). Actin dynamics plays an essential role in learning and memory. It has been demonstrated that synaptic remodeling mediated by actin rearrangements is important for consolidation and extinction of contextual fear conditioning in hippocampus (Fischer et al. 2004). In addition, consolidation of auditory fear conditioning depends on actin polymerization in the lateral

\footnotetext{
${ }^{1}$ Corresponding author.
}

E-mail zheyuchen@sdu.edu.cn; fax 86-531-88382329.

Article is online at http://www.learnmem.org/cgi/doi/10.1101//m.1772310. amygdala (Mantzur et al. 2009). Recent study supports that actin rearrangements in the amygdala and dorsal hippocampus are required for the acquisition and consolidation of the aversive memories of drug withdrawal (Hou et al. 2009).

However, the temporal and spatial regulation of actin dynamics-related synaptic structure in different phases of associative memory has not been fully understood. Therefore, in the present study using the CTA paradigm, an excellent model system for cortical learning, we try to address the temporal and spatial involvement of actin rearrangement-related synaptic structure alterations in different phases of CTA memory.

\section{Results}

Synapse alterations during CTA short-term memory formation

Actin rearrangement plays an important role in learning and memory through the formation of new synapses and remodeling of the existing ones. First, we try to examine synapse alterations in related brain regions (BLA, IC, and PrL) during CTA short-term memory formation using electron microscopy. To address this issue, rats were divided into five groups: the naïve, CS, US, CSUS-Long (CS-US-L) control, and CTA training groups. On the conditioning day, rats in the CTA training group were given saccharin for $10 \mathrm{~min}$ and intraperitoneally injected with lithium chloride (LiCl) $40 \mathrm{~min}$ later. For the naïve group, rats were only trained to get their daily water ration within $10 \mathrm{~min}$. In the CS group, rats were given saccharin only, without the following intraperitoneal injection of $\mathrm{LiCl}$. Rats in the US group were treated the same as the CTA training group, but instead of getting the CS, will get water in the training day. In the CS-US-L control group, rats received a $\mathrm{LiCl}$ injection $24 \mathrm{~h}$ after saccharin drinking, as long CS-US intervals could abolish the CTA learning (Gutierrez et al. 
2003). We chose a 4-h post-LiCl injection to test STM of taste aversion according to the previous report (Lamprecht et al. 1997). As shown in Figure 1A, post hoc comparisons revealed significant differences between the CTA training group and the naïve, CS, US, and CS-US-L control groups, demonstrating that the conditioned animals have acquired the short-term memory $(\mathrm{STM})\left(F_{(4,16)}=\right.$ 52.127, $P<0.01)$. The tissues of BLA, IC, and PrL were then dissected for electron microscopic (EM) analysis. Synaptic profiles (arrows) are defined by a presynaptic component with synaptic vesicles and a clearly defined postsynaptic density (PSD). The PSD is an organelle enriched in actin and contains a variety of molecules involved in signal transduction (Adam and Matus 1996). There were no obvious morphological changes of synapses in the BLA or PrL between the CTA training and control groups at $4 \mathrm{~h}$ after CTA training. However, increased vesicles assembled near the active zone of synapses were found in the IC of training group (Fig. 1B). More importantly, quantitative analysis revealed an increased PSD length in the IC $\left(F_{(4,13)}=12.185, P<0.01\right)$, but not in the BLA or PrL compared with other control groups during STM formation (Fig. 1C), which suggested that the synapse alterations during STM formation after CTA learning were due to the specific CS-US association, but not the CS or US stimulation alone. In contrast, the synapse density in the BLA, IC, and PrL did not change significantly compared with their control groups during STM formation (Fig. 1D). The CTA learning-induced PSD elongation in the IC during STM formation could be a morphological implication for enhanced synaptic efficacy (Geinisman et al. 2001; Nikonenko et al. 2002), which suggests that the actin dynamics in the IC may be involved in CTA STM formation.

\section{Synapse changes during CTA long-term memory formation}

Next, we investigated the synapse changes during CTA LTM formation in different brain regions. It is a general belief that the establishment of long-term memory (LTM) is associated with its long-lasting storage in synapses, which is accomplished either by an addition of new synaptic contacts or an increase in the strength of existing ones (Andersen and Soleng 1998; Klintsova and Greenough 1999; Geinisman 2000; Geinisman et al. 2001). LTM was tested at $72 \mathrm{~h}$ after $\mathrm{LiCl}$ injection according to a previous study (Lamprecht et al. 1997). As shown in Figure 2A, the aversion index in the CTA training group was significantly higher at $72 \mathrm{~h}$ post-LiCl injection compared with other control groups $\left(F_{(4,18)}=57.760, P<0.01\right)$, demonstrating that the conditioned animals have acquired LTM. The synapse changes in the BLA, IC, and PrL during CTA LTM formation were then examined by electron microscopy (Fig. 2B). Notably, at $72 \mathrm{~h}$ after CTA conditioning, post hoc comparisons revealed that both PSD length and synapse density in the IC (PSD length: $F_{(4,16)}=11.342, P<$ 0.01 ; synapse density: $F_{(4,17)}=3.887, P<0.05$ ) and PrL (PSD length: $F_{(4,16)}=8.997, P<0.01$; synapse density: $F_{(4,15)}=5.028$, $P<0.01$ ) were significantly increased during LTM formation compared with other control groups (Fig. 2C,D), which suggested that

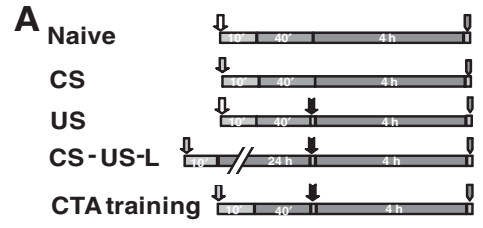

$\mathbb{n}$ water $\mathbb{}$ saccharin $\mathbb{L i C l} \mathbb{0}$ test

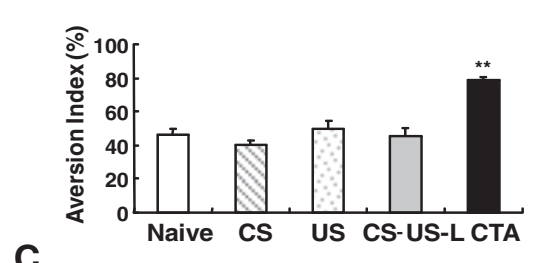

C

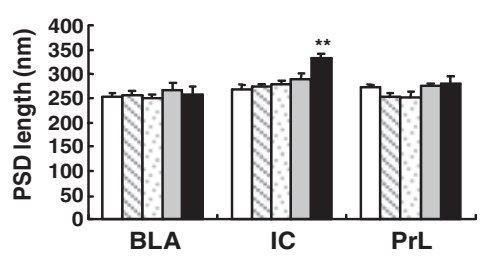

$D^{\infty}$

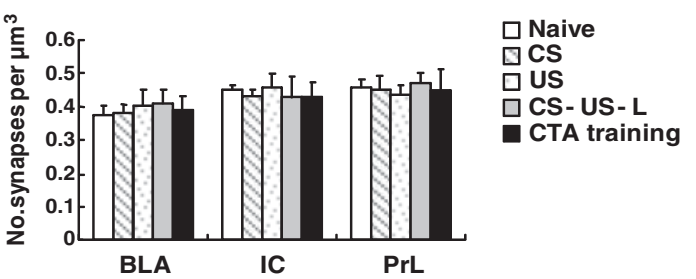

B

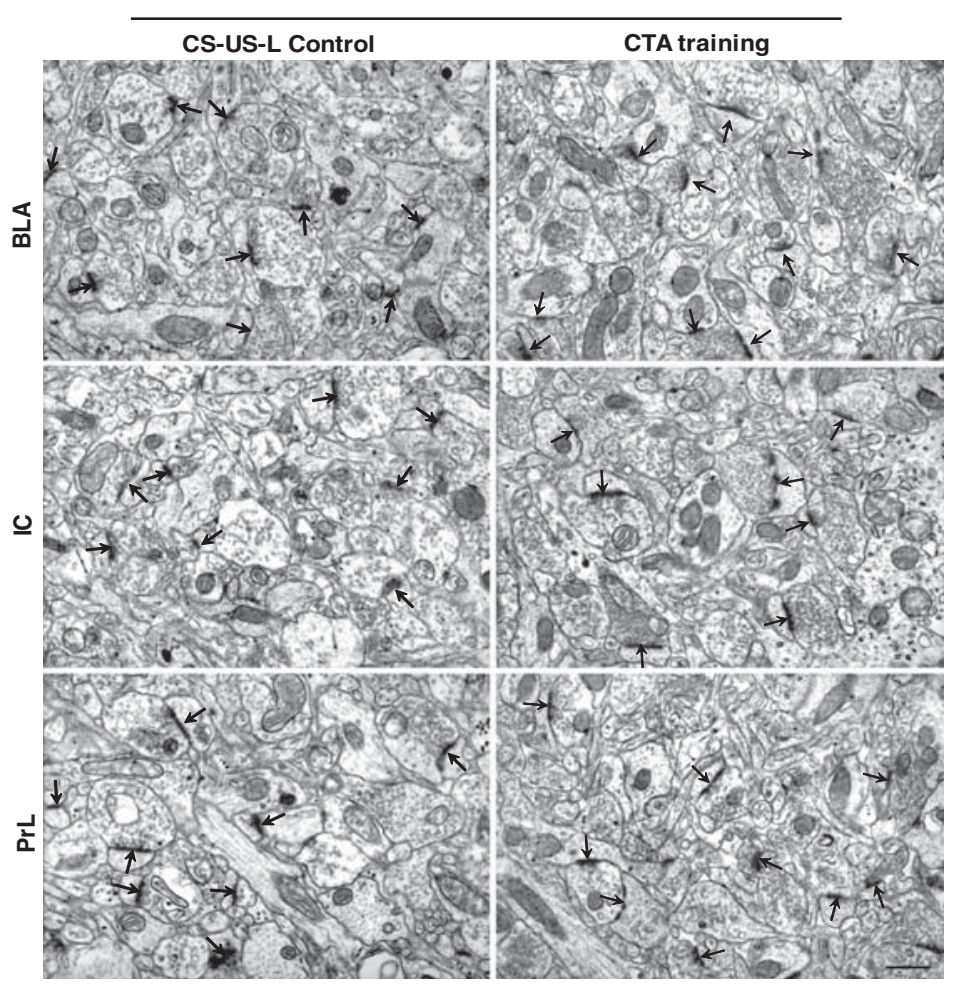

Figure 1. CTA increases PSD length in the IC during STM formation. ( $A$ ) STM formation after CTA conditioning. Schematic representation of the behavior protocol to evaluate CTA acquisition in the naïve, CS, US, CS-US-L control, and CTA training groups. The behavior tests were carried at $4 \mathrm{~h}$ after $\mathrm{LiCl}$ injection. (B) Representative electron micrographs show the synapses in the BLA, IC, and PrL of CS-US-L control and CTA training rats at $4 \mathrm{~h}$ after LiCI injection. Synapses are indicated by arrows. The scale bar represents $0.5 \mu \mathrm{m} .(C, D)$ Estimated PSD length (C) and synapse density $(D)$ in the BLA, IC, and PrL of the naïve, CS, US, CS-US-L control, and CTA training rats. All values represent means \pm SEM determined from analysis of four to six rats per group $\left({ }^{* *} P<0.01\right.$ compared with the naïve, CS, US, and CS-US-L control, respectively). 

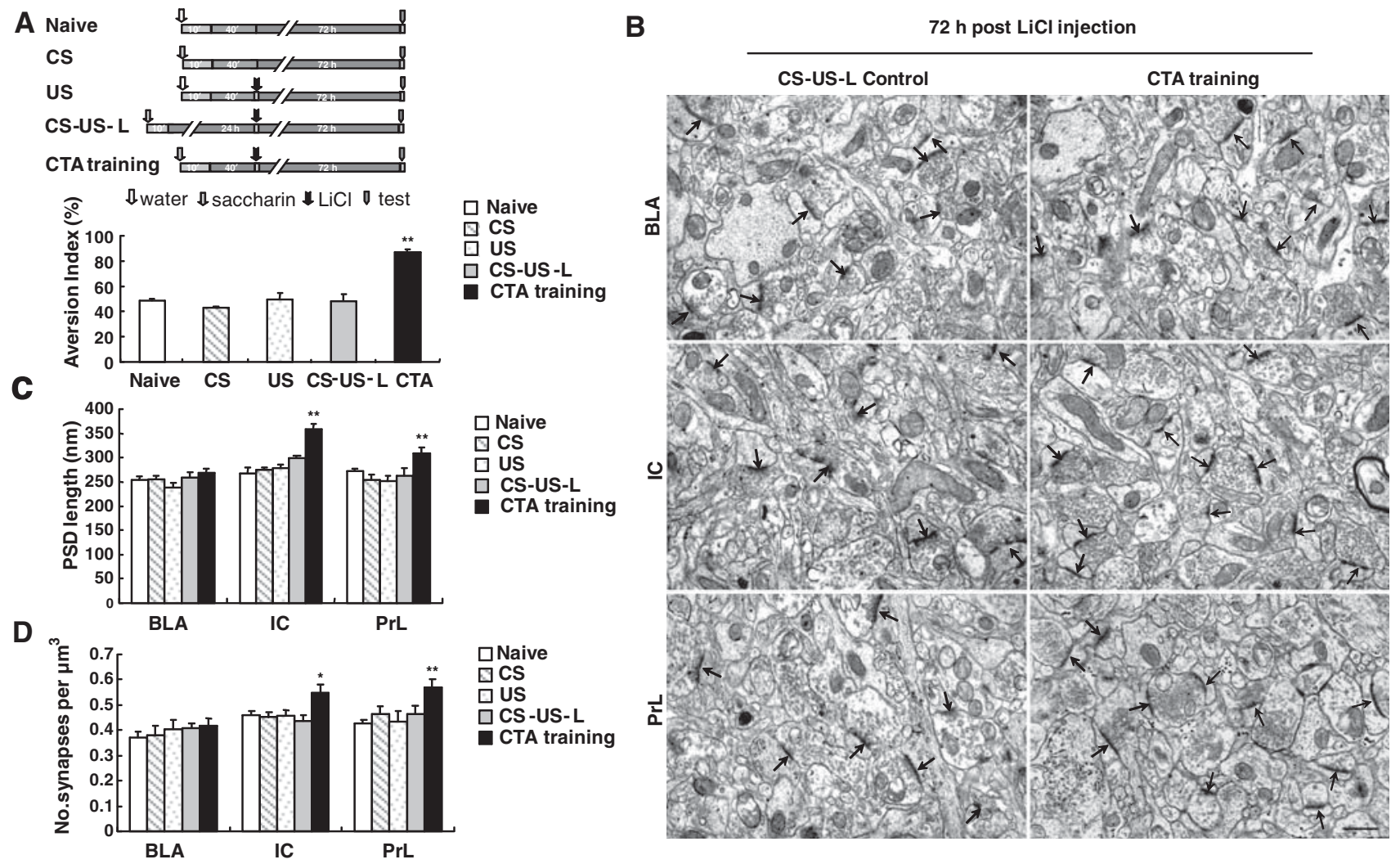

Figure 2. CTA increases PSD length and synapse density in the PrL and IC during LTM formation. ( $A$ ) LTM formation after CTA conditioning. Schematic representation of the behavior protocol to evaluate CTA acquisition in the naïve, CS, US, CS-US-L control, and CTA training groups. The behavior tests were carried out $72 \mathrm{~h}$ after LiCl injection. (B) Representative electron micrographs show the synapses in the BLA, IC, and PrL of CS-US-L Control and CTA training rats at $72 \mathrm{~h}$ after $\mathrm{LiCl}$ injection. Synapses are indicated by arrows. The scale bar represents $0.5 \mu \mathrm{m}$. $(C, D)$ Estimated PSD length $(C)$ and synapse density $(D)$ in the BLA, IC, and PrL of the naïve, CS, US, CS-US-L control, and CTA training rats. All values represent means \pm SEM determined from analysis of four to six rats per group $\left({ }^{*} P<0.05,{ }^{* *} P<0.01\right.$ compared with the naïve, CS, US, and CS-US-L control, respectively).

both the IC and PrL might be involved in CTA LTM formation. However, there were no significant changes in the synapse morphology and density in the BLA during LTM formation. Together with our previous results, we found PSD elongation in the IC during CTA STM formation, whereas CTA training could induce de novo synapse formation in the IC and PrL in addition to PSD elongation at a later time during LTM formation, which suggests distinct actin dynamic-related synaptic plasticity during STM and LTM formation of CTA memory.

\section{Actin rearrangement in the IC, but not in the BLA or PrL, is necessary for CTA acquisition}

Although the previous experiment demonstrated the increased PSD length within the IC during CTA STM formation, it is still unclear whether actin rearrangement is functionally necessary for CTA acquisition. To address this question, the inhibitor of actin polymerization was microinjected into the BLA, IC, or PrL 30 min before CTA training, and the aversion index was tested $4 \mathrm{~h}$ after conditioning (Fig. 3A). To exclude the nonspecific effect of the inhibitor, we chose two kinds of actin polymerization inhibitors (Latrunculin A and cytochalasin D) with different working mechanisms. Latrunculin A, which forms a nonpolymerizable 1:1 complex with G-actin, could sequester G-actin to inhibit actin polymerization, whereas cytochalasin D caps the growing end of actin filaments and thus changes the polymerization and depolymerization rates of F-actin (Chodniewicz et al. 2004). Injection of the inhibitors into the IC produced a significant reduction of the aversion index tested $4 \mathrm{~h}$ after training (Fig. 3B; $F_{(2,21)}=16.651, P<0.01$ ), indicating that administration of cytochalasin D or latrunculin A into the IC results in CTA acquisition impairment. On the contrary, when inhibitors were microinjected into the BLA or PrL, there were no significant differences of STM among the inhibitors and vehicle groups (Fig. 3B). Immediately after the STM test, the IC of the vehicle or cytochalasin D-treated group was dissected for synaptic morphology analysis by electron microscopy. As seen in Figure 3C, both the number of vesicles near the active zone and the PSD thickness decreased in the cytochalasin D-treated group. Quantitative analysis revealed that the cytochalasin D microinjection resulted in a decreased PSD length in the IC compared with the vehicle group (Fig. 3D; $t_{(7)}=3.904, P<0.01$ ). However, there was no significant difference in synapse density between the two groups (Fig. 3E). To exclude the possibility that the region-specific effect of actin polymerization inhibitor on CTA memory acquisition is due to the inhibitor's differential inhibition effect on actin polymerization in various areas, we microinjected vehicle or cytochalasin D into the BLA, IC, or PrL of naive rats and the corresponding brain regions were taken at $4 \mathrm{~h}$ after microinjection. Quantitative EM analysis revealed a decreased PSD length in the BLA, PrL, and IC (BLA, $t_{(4)}=2.987 ;$ PrL, $t_{(4)}=4.346$; IC, $t_{(4)}=$ 2.907, $P<0.05$ ) (Fig. 4A), but there were no significant changes in synapse density (Fig. 4B) in the cytochalasin D injection group, which suggested that cytochalasin $\mathrm{D}$ has a similar actin polymerization inhibition effect in various brain regions. The inhibition of actin rearrangement in the IC, but not in the BLA or PrL, 
A

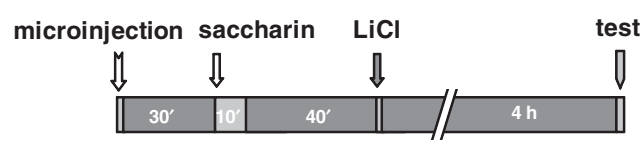

C

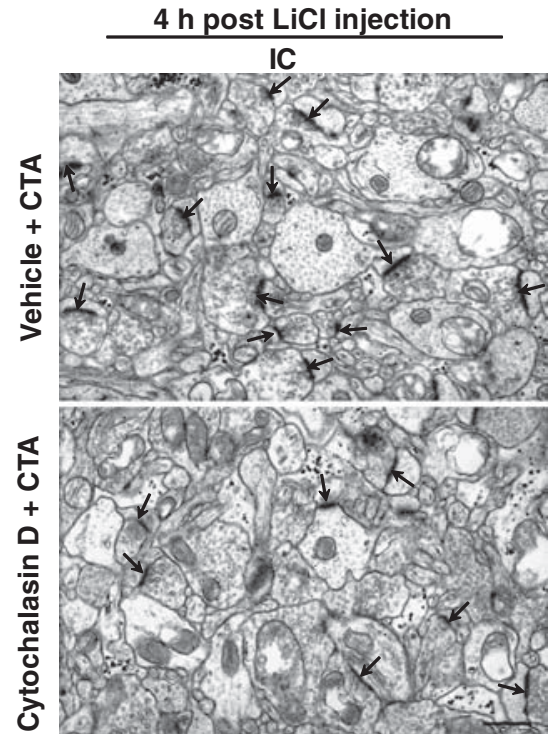

B

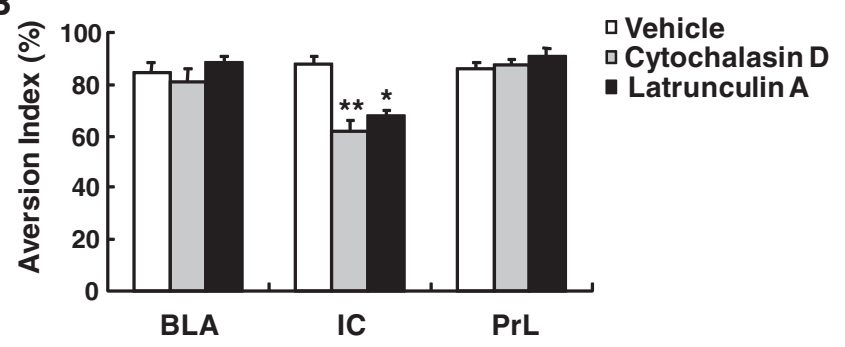

D

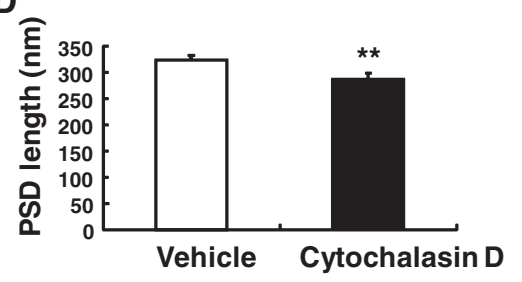

E

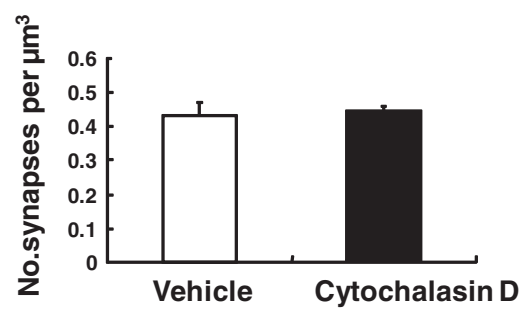

Figure 3. Microinjection of cytochalasin $D$ or latrunculin $A$ into the $I C$ before conditioning impairs CTA acquisition. (A) Schematic representation of the behavior protocol to evaluate CTA acquisition. (B) Microinjection of cytochalasin D, latrunculin $A$, or vehicle bilaterally into the BLA, IC, or PrL at 30 min before CTA training, and the aversion index were tested at 4-h post-conditioning. The values represent means \pm SEM determined from analysis of eight to 10 rats per group $\left({ }^{*} P<0.05,{ }^{* *} P<0.01\right.$ compared with vehicle). (C) Representative electron micrographs to illustrate the synapses in the IC of vehicle or cytochalasin D-treated rats. Synapses are indicated by arrows. The scale bar represents $0.5 \mu \mathrm{m}$. $(D, E)$ Estimated PSD length $(D)$ and synapse density $(E)$ in the IC of vehicle or cytochalasin D-treated rats. All values represent means \pm SEM determined from analysis of four to six rats per group ( ${ }^{* *} P<0.01$ compared with vehicle).

produces impairment in CTA memory acquisition, and its related synaptic plasticity suggested the involvement of actin dynamics within the IC during CTA memory acquisition.

\section{Actin rearrangements in the IC and PrL are required in CTA consolidation but not retrieval}

Since our previous results have shown that different synaptic changes occurred during LTM formation compared with the
STM formation period, we next investigated the functional role of spatial actin rearrangements in CTA consolidation, because the formation of LTM depends on memory consolidation, a progressive post-acquisition stabilization process. To ensure that the rats of each group acquire similar STM of CTA, cytochalasin $\mathrm{D}$ or latrunculin A was infused $4 \mathrm{~h}$ after conditioning and LTM was tested 72-h post-conditioning to measure the specific effect of actin dynamics on memory consolidation (Fig. 5A). Significant differences in aversion index were found among
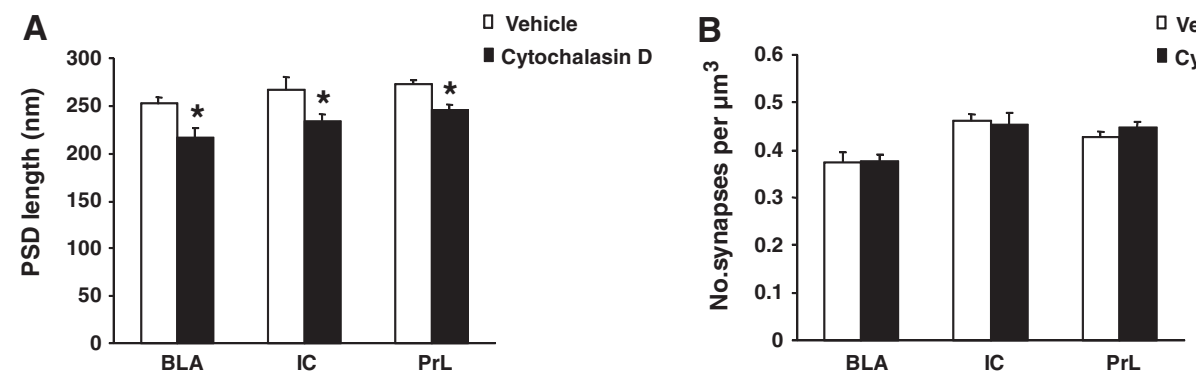

Figure 4. The effect of cytochalasin $D$ on actin polymerization in various brain areas. Microinjection of cytochalasin $D$ or vehicle into the BLA, IC, or PrL and the corresponding brain regions were taken at $4 \mathrm{~h}$ post-microinjection. (A) Estimated PSD length in the BLA, IC, and PrL of the vehicle and cytochalasin D microinjection group. (B) Estimated synapse density in the BLA, IC, and PrL of the vehicle and cytochalasin D microinjection group. All values represent means \pm SEM determined from analysis of three rats per group $\left({ }^{*} P<0.05\right.$ compared with vehicle). 


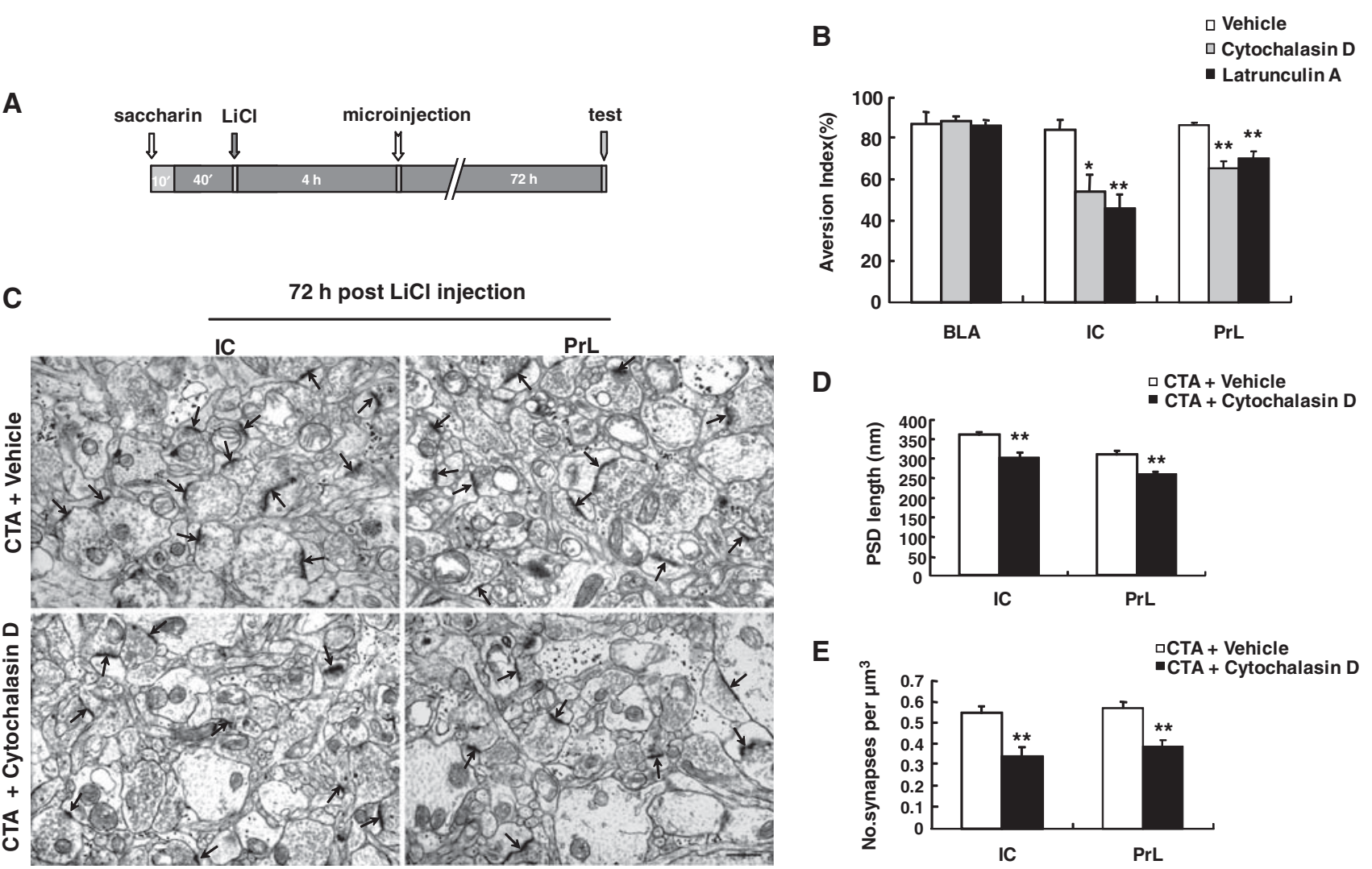

Figure 5. Microinjection of cytochalasin $D$ or latrunculin $A$ into the IC or PrL after training impairs CTA LTM. (A) Schematic representation of the behavior protocol to evaluate CTA consolidation. (B) Microinjection of cytochalasin D, latrunculin A, or vehicle bilaterally into the BLA, IC, or PrL at $4 \mathrm{~h}$ after CTA training and the aversion index were tested at 72-h post-conditioning. The values represent means \pm SEM determined from analysis of eight to 10 rats per group $\left({ }^{*} P<0.05,{ }^{*} P<0.01\right.$ compared with vehicle). (C) Representative electron micrographs to illustrate the synapses in the IC and PrL of vehicle or cytochalasin $D$-treated rats. Synapses are indicated by arrows. The scale bar represents $0.5 \mu \mathrm{m}$. $(D, E)$ Estimated PSD length $(D)$ and synapse density $(E)$ in the IC and PrL of vehicle or cytochalasin D-treated rats. All values represent means \pm SEM determined from analysis of four to six rats per group $\left({ }^{* *} P<0.01\right.$ compared with vehicle).

the groups of IC injection $\left(F_{(2,23)}=33.125, P<0.01\right)$ or PrL injection $\left(F_{(2,27)}=33.605, P<0.01\right)$ (Fig. 5B). However, microinjection of cytochalasin D or latrunculin A into the BLA has no effect on the formation of CTA LTM. These results demonstrate that actin dynamics in the IC and PrL but not in the BLA are required for CTA LTM formation. Following the LTM test, the IC and PrL of the vehicle and cytochalasin D-treated groups were dissected for electron microscopy analysis (Fig. 5C). Quantitative analysis revealed that there was significant decreased synapse density and reduced PSD length in the cytochalasin D group compared with the vehicle group in the IC (synapse density: $t_{(6)}=4.251$, $P<0.01$; PSD length: $t_{(7)}=4.404, P<0.01$ ) and PrL (synapse density: $t_{(7)}=4.338, P<0.01$; PSD length: $t_{(7)}=4.027, P<0.01$ ), which were in accordance with the behavioral results (Fig. 5D,E).

LTM impairment could also be due to memory retrieval deficit. In an additional experiment, we microinjected cytochalasin D or latrunculin A into the BLA, IC, or PrL 30 min before LTM test (Fig. 6A). All groups were able to acquire similar levels of LTM compared with vehicle group (Fig. 6B). Thus, inhibition of actin rearrangement in the BLA, IC, or PrL did not interfere with the animals' ability to retrieve acquired CTA memory. In all, these results demonstrate that blocking of actin dynamics in the IC or PrL produces impairment in CTA memory consolidation but not in memory retrieval, implying that there are different roles for actin rearrangement in these two fundamental processes of memory.

\section{Discussion}

There are few reports about the role of actin rearrangement in CTA memory processes. In this study, we provide evidence for the first time that actin rearrangement-related synapse alterations play important roles in CTA. Our data provide several new insights into the region-specific involvement of actin dynamics-related synaptic structure changes in different phases of CTA memory. First, we found different synaptic plasticity during STM and LTM formation of CTA. During CTA STM formation, a structural remodeling of existing synapses occurred in the IC, resulting in an enlargement of the PSD that depends on actin cytoskeleton rearrangement, whereas in addition to the increase of existing synapse strength, during LTM establishment there is formation of new synapses in the IC and PrL that are required for the maintenance of CTA memory. These findings suggest that there are temporal regulations of actin rearrangement-related synaptic plasticity in different brain regions during CTA memory processes.

Second, we found the region-specific involvement of actin dynamics in CTA memory processes. Previous studies have shown that CTA can be elicited by intra-BLA microinjections of glutamate (Miranda et al. 2002) and post-training infusion of corticosterone into the BLA could enhance CTA memory retention (Miranda et al. 2008), which indicates that intra-BLA neurotransmission is required for CTA memory. However, we found actin 

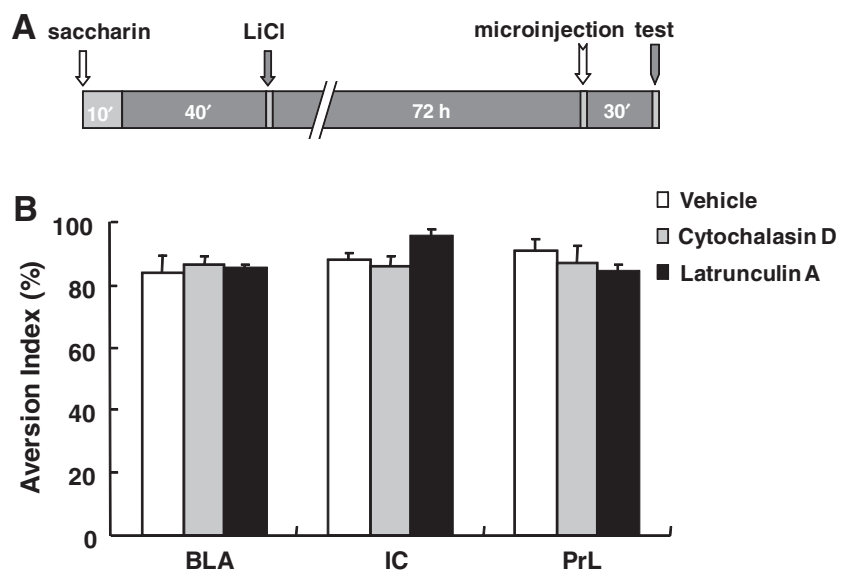

Figure 6. Microinjection of cytochalasin $D$ or latrunculin $A$ into the BLA, IC, or PrL before LTM test has no effect on CTA memory retrieval. (A) Schematic representation of the behavior protocol to evaluate CTA retrieval. (B) Microinjection of cytochalasin D, latrunculin A, or vehicle bilaterally into the BLA, IC, or PrL at 30 min before the LTM test and the aversion Index were tested at 72-h post-conditioning. All values represent means \pm SEM determined from analysis of eight to 10 rats per group.

rearrangement in the BLA does not participate in CTA acquisition and consolidation, which suggests that the actin dynamics and neurotransmission are two distinct mechanisms underlying memory formation. Our data is consistent with a previous report that local blockade of protein synthesis in the BLA did not disrupt CTA memory formation (Bahar et al. 2003, 2004). Interestingly, actin cytoskeleton rearrangement in the BLA was reported to be essential for the consolidation of auditory fear conditioning (Mantzur et al. 2009), which suggests the selective involvement of actin dynamics in different behavior tests.

Pharmacological manipulation experiments demonstrate that insular cortical muscarinic and NMDA receptors and mitogen-activated protein kinase cascades are involved in CTA acquisition and consolidation (Rosenblum et al. 1997; Berman et al. 1998, 2000; Ferreira et al. 2002; Gutierrez et al. 2003). Our data further suggest that actin rearrangement in the IC is also necessary for these CTA memory processes; however, its role is not indispensable, as animals can still acquire memory to a lesser extent after inhibition of actin dynamics in the IC. Earlier study suggests that activity in the PrL is critical for the consolidation, but not the acquisition, of auditory or contextual fear memories (Corcoran and Quirk 2007). In trace eyeblink conditioning learning it has been shown that medial prefrontal cortex (including PrL) is essential for remotely, but not recently acquired memory (Takehara et al. 2003). Furthermore, Tronel and coworkers found that the noradrenergic system in the PrL was required for the late stage of memory consolidation in an appetitively motivated foraging task (Tronel et al. 2004). Consistent with these behavior tests, the present study has demonstrated that actin rearrangement in the PrL is required for the consolidation of CTA. Interestingly, our findings also imply that actin rearrangements in the PrL and IC play different roles in CTA memory. As in the PrL, actin dynamics is not necessary for the acquisition of shortterm CTA memory but is required for LTM formation. Since previous study has shown that the PrL and IC are interconnected (Gabbott et al. 2003), it is possible that the PrL plays a special role in organizing the consolidation of CTA memory in the IC.

Finally, our behavioral experiments revealed that actin rearrangement in the BLA, IC, or PrL is not essential in CTA memory retrieval, which indicates that the cellular mechanisms of memory acquisition and consolidation are different from memory retrieval. It has been reported that pretest administration of NMDA receptor antagonists, at doses that blocked long-term spatial memory formation did not alter memory retrieval (Steele and Morris 1999; Szapiro et al. 2000). On the other hand, in a foodmotivated maze task, the memory retrieval can be enhanced by the activation of $\beta$-adrenergic receptors (Sara and Devauges 1988; Devauges and Sara 1991). In addition, in a hippocampaldependent inhibitory avoidance task, memory retrieval can be enhanced by AMPA and metabotropic glutamate receptor activation (Szapiro et al. 2002). However, actin rearrangement is not involved in memory retrieval process, at least not in CTA memory.

In summary, we used a transmission electron microscope to demonstrate distinct synaptic alterations during short-term and long-term CTA memory. We found the brain region-specific involvement of actin dynamics in CTA memory processes. Actin rearrangement in the PrL is necessary for memory consolidation, and in the IC it is required for both the acquisition and consolidation of CTA memory. In addition, our results also imply that actin dynamics plays different roles in different phases of CTA memory. Both CTA memory acquisition and consolidation depend on actin rearrangements, whereas actin dynamics is not involved in the retrieval process. The detailed mechanisms of actin dynamics during CTA memory need to be investigated in the future.

\section{Materials and Methods}

\section{Subjects}

The subjects were male Wistar rats (60-d-old, 250-280 g), caged individually at $22^{\circ} \mathrm{C} \pm 2^{\circ} \mathrm{C}$ under 12 -h light/12-h dark cycles. Water and food were available ad libitum except when experimental requirements dictated otherwise. All procedures were in accordance with the NIH Guide for the Care and Use of Laboratory Animals and were approved by the Institutional Animal Care and Use Committee of Shandong University.

\section{Reagent}

Latrunculin A and cytochalasin D were purchased from Sigma and dissolved in ACSF $\left(124 \mathrm{mM} \mathrm{NaCl}, 3 \mathrm{mM} \mathrm{KCl}, 1.25 \mathrm{mM} \mathrm{KH}_{2} \mathrm{PO}_{4}\right.$, $1 \mathrm{mM} \mathrm{MgSO} 4 \cdot 7 \mathrm{H}_{2} \mathrm{O}, 26 \mathrm{mM} \mathrm{NaHCO} 3,10 \mathrm{mM}$ D-glucose, $2 \mathrm{mM}$ $\mathrm{CaCl}_{2} \cdot 2 \mathrm{H}_{2} \mathrm{O}$ at $\mathrm{pH}$ 7.4) containing $1 \% \mathrm{DMSO}$ to a final concentration of $500 \mathrm{ng} / \mu \mathrm{L}$.

\section{Behavioral procedures}

In the CTA procedure, saccharin $(0.1 \% \mathrm{w} / \mathrm{v}$, sodium salt, Sigma Chemical Co.) was used as an unfamiliar taste, and intraperitoneal $\mathrm{LiCl}(0.15 \mathrm{M}, 2 \%$ body weight, Sigma-Aldrich Chemical Co.) was used as a malaise-inducing agent. The CTA protocol was essentially as described by a previous report (Rosenblum et al. 1993). In brief, rats were trained over $4 \mathrm{~d}$ to get their daily water ration within $10 \mathrm{~min}$ from two pipettes, each containing $10 \mathrm{~mL}$ of tap water. On day 5 (conditioning day), water was replaced with tastant solution (5 $\mathrm{mL}$ of $0.1 \%$ saccharin). This was followed $40 \mathrm{~min}$ later by an intraperitoneal injection of $\mathrm{LiCl}$. For the naïve group, rats were only trained to get their daily water ration within $10 \mathrm{~min}$. In the CS group, rats were given saccharin only, without the following intraperitoneal injection of LiCl. Rats in the US group were treated the same as the CTA training group, but instead of getting the CS will get water on the training day. In the CS-US-L control group, rats received a $\mathrm{LiCl}$ injection $24 \mathrm{~h}$ after drinking saccharin. Four or $72 \mathrm{~h}$ after the injection of $\mathrm{LiCl}$ rats were presented with an array of six pipettes, three containing $5 \mathrm{~mL}$ of saccharin and three containing $5 \mathrm{~mL}$ of water, for $10 \mathrm{~min}$, and their liquid consumption was recorded to test the acquisition of short-term and long-term CTA memory, respectively. The aversion index (AI) was defined as (milliliters of water/[milliliters of water + milliliters of saccharin] $100 \%$ consumed in the test. In 
STM measurement, inhibitor or vehicle was microinjected into different brain regions at $30 \mathrm{~min}$ before training, and AI were tested at $4 \mathrm{~h}$ after conditioning to measure memory acquisition. For LTM measurement, inhibitor or vehicle was microinjected at $4 \mathrm{~h}$ after conditioning and AI were tested at $72 \mathrm{~h}$ after conditioning. To evaluate memory retrieval, inhibitor or vehicle was microinfused at $30 \mathrm{~min}$ before LTM test ( $72 \mathrm{~h}$ after conditioning). Rats were randomly assigned to receive either vehicle, cytochala$\sin \mathrm{D}$, or latrunculin A microinjection ( $n=8-10$ for each group).

\section{Surgery and microinjection}

Microinjection was performed via chronically implanted cannulas. Rats were anesthetized with $5 \%$ chloral hydrate $(0.6 \mathrm{~mL} /$ $100 \mathrm{~g}$ ), restrained in a stereotaxic apparatus (Reward 8001), and implanted bilaterally with guide cannulas (stainless steel, 23 gauge) aimed at the PrL, BLA, or IC (coordinates in reference to bregma, PrL: anteroposterior [AP], $+3.15 \mathrm{~mm}$; lateral [L], $\pm 0.5 \mathrm{~mm}$; and ventral [V], $-2.7 \mathrm{~mm}$. BLA: AP, $-2.8 \mathrm{~mm}$; $\mathrm{L}$, $\pm 4.8 \mathrm{~mm}, \mathrm{~V},-6.7 \mathrm{~mm}$. IC: $\mathrm{AP},+1.3 \mathrm{~mm} ; \mathrm{L}, \pm 0.3 \mathrm{~mm}$; V, $-4.5 \mathrm{~mm}$ ) (Paxinos and Watson 1996). A stylus was placed in the guide cannula to prevent clogging. The injection cannula was extended $1.5 \mathrm{~mm}$ from the tip of the guide cannula. Animals were allowed $1 \mathrm{wk}$ to recuperate before being subjected to experimental manipulations. Microinjection of cytochalasin D or latrunculin A (500 ng in $1 \mu \mathrm{L} /$ hemisphere) was performed at the times indicated in Behavioral Procedures. The stylus was removed from the guide cannula, and a 28-gauge injection cannula was carefully inserted. The injection cannula was connected via PE20 tubing to a Hamilton microsyringe driven by a microinjection pump (Carnegie Medicine CMA 100) at a rate of $1.0 \mu \mathrm{L} /$ min. After microinjection, the injection cannula was left for an additional $2 \mathrm{~min}$ before withdrawal to reduce efflux of injection liquid along the injection tract.

\section{Histology}

Following completion of the experimental protocol rats were deeply anaesthetized and $1 \mu \mathrm{L}$ of methylene blue was microinfused. Frozen brain slices $(40 \mu \mathrm{m})$ were analyzed to verify the microinfusion sites and the solution diffused scope in the BLA, IC, and PrL (Supplemental Fig. S1).

\section{Tissue preparation for electron microscopy}

To accurately locate the tiny electron microscope (EM) sample for analysis, we applied a three-step localization method (Supplemental Fig. S2). Rats were deeply anesthetized with 5\% chloral hydrate $(0.6 \mathrm{~mL} / 100 \mathrm{~g})$ and perfused with $100-200 \mathrm{~mL}$ of $0.9 \%$ sodium chloride $(\mathrm{pH} 7.4)$, followed by $100-200 \mathrm{~mL}$ phosphate-buffered fixative ( $\mathrm{pH}$ 7.4) containing $2 \%$ paraformaldehyde and $2.5 \%$ glutaraldehyde. Brains were rapidly removed, put in a matrix surrounded by cold ice and cut into 1-mm-thick slices. The first step is anatomical localization. In accordance with the atlas (Paxinos and Watson 1996), the slice containing the BLA, IC, or PrL was cut into a cuboid block (Supplemental Fig. S2A,B show the PrL). In order to make the estimation of electron microscopy objective, each block of the BLA, PrL, or IC was then divided into three $500-\mu \mathrm{m}$-thick slices. The slices were postfixed in $2.5 \%$ glutaraldehyde in $0.1 \mathrm{M}$ phosphate-buffered saline for $4 \mathrm{~h}$, followed by $1 \%$ osmium tetroxide treatment for $1.5 \mathrm{~h}$, dehydrated, and the 500- $\mu$ m-thick slices were then embedded in Epon 812. Secondly, we applied histological localization on the semithin sections of the EM sample. As the pyramidal cells in the prefrontal cortex play important roles in the learning and memory processes (Degenetais et al. 2003), and in addition the cortex layer $\mathrm{V}$ is adjacent to the white matter and is easy for localization, we focused on the research of synapses in the cortex lamina V. For each embedded slice, a 2 - $\mu$ m-thick semithin section was cut and stained with hematoxylin and eosine for localization. The cortex layer $\mathrm{V} / \mathrm{VI}$ and the white matter can be observed in the semithin section (Supplemental Fig. S2C). According to the localization of the semithin section, the embedded slice of the PrL or
IC was then trimmed to contain only cortex layer V/VI. The embedded slices of BLA that only contain nerve fibers were excluded from the following experiment. The third step is ultrastructural localization. The serially $65-\mathrm{nm}$-thick ultrathin sections were then cut and collected on formvar-coated, single-slot grids, stained with uranyl acetate and lead citrate, and viewed on a JEM-1200 EX electron microscope. A single-slot grid was collected per embedded slice, and totally, three single-slot grids were collected per block. The lamina V/VI is easily identified at low magnification under the electron microscope and the big pyramidal cells have distinct ultrastructural characteristics. The visual field of the PrL or IC for photographing was chosen strictly around big pyramidal neurons in layer V (Supplemental Fig. S2D,E shows an ultrathin section of the PrL).

\section{Data analysis for electron micrographs}

The density of synapses per unit volume $\left(\mu \mathrm{m}^{3}\right)$ was estimated on electron micrographs from rats of different experimental conditions in a double blind fashion using the conventional method, that is, using the formula $N_{\mathrm{V}}=N_{\mathrm{A}} / d$, where $N_{\mathrm{A}}$ is the number of synaptic profiles per unit area and $d$ is the average cross-sectional length of synaptic junctions (Beaulieu and Colonnier 1985; Alonso-Nanclares et al. 2008). For each block (totally three singleslot grids), 15 noncontiguous visual fields at the magnification of 5000 , covering neuropil regions totaling $3000 \mu \mathrm{m}^{2}$ were used for quantitation. Notably, photographs of the PrL or IC were taken randomly around the big pyramidal cells in pyramidal cell layer V. Regions containing cell bodies, myelinated processes, or blood vessels were intentionally avoided. The synapses are defined as having a presynaptic membrane, a postsynaptic density, and vesicles in the presynaptic terminal. Perforated synaptic junctions were treated as single synapse. The detailed estimation of synapse was described in the previous report (DeFelipe et al. 1999). The number of synapses, exact areas of the visual fields, and the crosssectional length of synaptic junctions $(d)$ were determined on electron micrographs using Metamorph software. For the determination of the total synapse $/ \mu \mathrm{m}^{3}$ and PSD length, the average of 15 visual fields of each animal was derived and expressed as mean per animal. There are four to six animals per group.

\section{Statistical analyses}

Data were analyzed with a Student's $t$-test and one-way ANOVA, followed by Fisher's LSD post hoc comparisons, where appropriate. The significance level was set to 0.05 for all statistical analyses, and all values in the text and figures represent means \pm SEM. Data analyses were performed using SPSS statistical program version 10.0 .

\section{Acknowledgments}

This study was supported by the National Natural Science Foundation of China (nos. 30725020, 30700258, 90713016), the National 973 Basic Research Program of China (nos. 2009CB941403, 2009CB526507), Science and Technology Development Projects of Shandong Province (no. 2008GG30002039), Fok Ying Tong Education Foundation (no.111044), Shandong Province Natural Science Foundation (no. Y2008D19), and the Foundation for Excellent Young Scientists of Shandong Province (no. BS2009SW028).

\section{References}

Adam G, Matus A. 1996. Role of actin in the organisation of brain postsynaptic densities. Brain Res Mol Brain Res 43: 246-250.

Akirav I, Khatsrinov V, Vouimba RM, Merhav M, Ferreira G, Rosenblum K, Maroun M. 2006. Extinction of conditioned taste aversion depends on functional protein synthesis but not on NMDA receptor activation in the ventromedial prefrontal cortex. Learn Mem 13: 254-258.

Alonso-Nanclares L, Gonzalez-Soriano J, Rodriguez JR, DeFelipe J. 2008. Gender differences in human cortical synaptic density. Proc Natl Acad Sci 105: 14615-14619. 
Andersen P, Soleng AF. 1998. Long-term potentiation and spatial training are both associated with the generation of new excitatory synapses. Brain Res Brain Res Rev 26: 353-359.

Bahar A, Samuel A, Hazvi S, Dudai Y. 2003. The amygdalar circuit that acquires taste aversion memory differs from the circuit that extinguishes it. Eur J Neurosci 17: 1527-1530.

Bahar A, Dorfman N, Dudai Y. 2004. Amygdalar circuits required for either consolidation or extinction of taste aversion memory are not required for reconsolidation. Eur J Neurosci 19: 1115-1118.

Beaulieu C, Colonnier M. 1985. A laminar analysis of the number of round-asymmetrical and flat-symmetrical synapses on spines, dendritic trunks, and cell bodies in area 17 of the cat. J Comp Neurol 231: $180-189$.

Berman DE, Hazvi S, Rosenblum K, Seger R, Dudai Y. 1998. Specific and differential activation of mitogen-activated protein kinase cascades by unfamiliar taste in the insular cortex of the behaving rat. J Neurosci 18: 10037-10044.

Berman DE, Hazvi S, Neduva V, Dudai Y. 2000. The role of identified neurotransmitter systems in the response of insular cortex to unfamiliar taste: Activation of ERK1-2 and formation of a memory trace. J Neurosci 20: 7017-7023.

Bliss TV, Collingridge GL. 1993. A synaptic model of memory: Long-term potentiation in the hippocampus. Nature 361: 31-39.

Buchs PA, Muller D. 1996. Induction of long-term potentiation is associated with major ultrastructural changes of activated synapses. Proc Natl Acad Sci 93: 8040-8045.

Bures J, Buresova O, Kriveanek J. 1988. Brain and behavior: Paradigms for research on neuronal mechanisms. Wiley, New York.

Chodniewicz D, Alteraifi AM, Zhelev DV. 2004. Experimental evidence for the limiting role of enzymatic reactions in chemoattractant-induced pseudopod extension in human neutrophils. J Biol Chem 279: 24460-24466.

Corcoran KA, Quirk GJ. 2007. Activity in prelimbic cortex is necessary for the expression of learned, but not innate, fears. J Neurosci 27: 840-844.

Cui Z, Lindl KA, Mei B, Zhang S, Tsien JZ. 2005. Requirement of NMDA receptor reactivation for consolidation and storage of nondeclarative taste memory revealed by inducible NR1 knockout. Eur I Neurosci 22: $755-763$.

DeFelipe J, Marco P, Busturia I, Merchan-Perez A. 1999. Estimation of the number of synapses in the cerebral cortex: Methodological considerations. Cereb Cortex 9: 722-732.

Degenetais E, Thierry AM, Glowinski J, Gioanni Y. 2003. Synaptic influence of hippocampus on pyramidal cells of the rat prefrontal cortex: An in vivo intracellular recording study. Cereb Cortex 13: 782-792.

Devauges V, Sara SJ. 1991. Memory retrieval enhancement by locus coeruleus stimulation: Evidence for mediation by $\beta$-receptors. Behav Brain Res 43: 93-97.

Ferreira G, Gutierrez R, De La Cruz V, Bermudez-Rattoni F. 2002. Differential involvement of cortical muscarinic and NMDA receptors in short- and long-term taste aversion memory. Eur J Neurosci 16: $1139-1145$.

Fifkova E, Morales M. 1992. Actin matrix of dendritic spines, synaptic plasticity, and long-term potentiation. Int Rev Cytol 139: 267-307.

Fischer A, Sananbenesi F, Schrick C, Spiess J, Radulovic J. 2004. Distinct roles of hippocampal de novo protein synthesis and actin rearrangement in extinction of contextual fear. J Neurosci 24: $1962-1966$

Fukazawa Y, Saitoh Y, Ozawa F, Ohta Y, Mizuno K, Inokuchi K. 2003. Hippocampal LTP is accompanied by enhanced F-actin content within the dendritic spine that is essential for late LTP maintenance in vivo. Neuron 38: 447-460.

Gabbott PL, Warner TA, Jays PR, Bacon SJ. 2003. Areal and synaptic interconnectivity of prelimbic (area 32), infralimbic (area 25) and insular cortices in the rat. Brain Res 993: 59-71.

Geinisman Y. 2000. Structural synaptic modifications associated with hippocampal LTP and behavioral learning. Cereb Cortex 10: 952-962.

Geinisman Y, Berry RW, Disterhoft JF, Power JM, Van der Zee EA. 2001. Associative learning elicits the formation of multiple-synapse boutons. J Neurosci 21: 5568-5573.
Gutierrez R, Rodriguez-Ortiz CJ, De La Cruz V, Nunez-Jaramillo L, Bermudez-Rattoni F. 2003. Cholinergic dependence of taste memory formation: Evidence of two distinct processes. Neurobiol Learn Mem 80: $323-331$.

Hou YY, Lu B, Li M, Liu Y, Chen J, Chi ZQ, Liu JG. 2009. Involvement of actin rearrangements within the amygdala and the dorsal hippocampus in aversive memories of drug withdrawal in acute morphine-dependent rats. J Neurosci 29: 12244-12254.

Houpt TA, Philopena JM, Joh TH, Smith GP. 1996. c-Fos induction in the rat nucleus of the solitary tract correlates with the retention and forgetting of a conditioned taste aversion. Learn Mem 3: 25-30.

Klintsova AY, Greenough WT. 1999. Synaptic plasticity in cortical systems. Curr Opin Neurobiol 9: 203-208.

Lamprecht R, Hazvi S, Dudai Y. 1997. cAMP response element-binding protein in the amygdala is required for long- but not short-term conditioned taste aversion memory. J Neurosci 17: 8443-8450.

Maletic-Savatic M, Malinow R, Svoboda K. 1999. Rapid dendritic morphogenesis in CA1 hippocampal dendrites induced by synaptic activity. Science 283: 1923-1927.

Mantzur L, Joels G, Lamprecht R. 2009. Actin polymerization in lateral amygdala is essential for fear memory formation. Neurobiol Learn Mem 91: $85-88$.

Miranda MI, Ferreira G, Ramirez-Lugo L, Bermudez-Rattoni F. 2002. Glutamatergic activity in the amygdala signals visceral input during taste memory formation. Proc Natl Acad Sci 99: 11417-11422.

Miranda MI, Quirarte GL, Rodriguez-Garcia G, McGaugh JL, Roozendaal B. 2008. Glucocorticoids enhance taste aversion memory via actions in the insular cortex and basolateral amygdala. Learn Mem 15: $468-476$.

Nikonenko I, Jourdain P, Alberi S, Toni N, Muller D. 2002. Activity-induced changes of spine morphology. Hippocampus 12: 585-591.

Norgren R. 1995. The rat nervous system (ed. G Paxinos). Academic Press, San Diego.

Paxinos G, Watson C. 1996. The rat brain in stereotaxic coordinates. Academic Press, New York.

Rosenblum K, Meiri N, Dudai Y. 1993. Taste memory: The role of protein synthesis in gustatory cortex. Behav Neural Biol 59: 49-56.

Rosenblum K, Berman DE, Hazvi S, Lamprecht R, Dudai Y. 1997. NMDA receptor and the tyrosine phosphorylation of its $2 \mathrm{~B}$ subunit in taste learning in the rat insular cortex. J Neurosci 17: $5129-5135$.

Sara SJ, Devauges V. 1988. Priming stimulation of locus coeruleus facilitates memory retrieval in the rat. Brain Res 438: 299-303.

Steele RJ, Morris RG. 1999. Delay-dependent impairment of a matching-to-place task with chronic and intrahippocampal infusion of the NMDA-antagonist D-AP5. Hippocampus 9: 118-136.

Szapiro G, Izquierdo LA, Alonso M, Barros D, Paratcha G, Ardenghi P, Pereira P, Medina JH, Izquierdo I. 2000. Participation of hippocampal metabotropic glutamate receptors, protein kinase A and mitogen-activated protein kinases in memory retrieval. Neuroscience 99: $1-5$.

Szapiro G, Galante JM, Barros DM, Levi de Stein M, Vianna MR, Izquierdo LA, Izquierdo I, Medina JH. 2002. Molecular mechanisms of memory retrieval. Neurochem Res 27: 1491-1498.

Takehara K, Kawahara S, Kirino Y. 2003. Time-dependent reorganization of the brain components underlying memory retention in trace eyeblink conditioning. J Neurosci 23: 9897-9905.

Tronel S, Feenstra MG, Sara SJ. 2004. Noradrenergic action in prefrontal cortex in the late stage of memory consolidation. Learn Mem 11: $453-458$.

Yu H, Wang Y, Pattwell S, Jing D, Liu T, Zhang Y, Bath KG, Lee FS, Chen ZY. 2009. Variant BDNF Val66Met polymorphism affects extinction of conditioned aversive memory. J Neurosci 29: 4056-4064.

Zhou Q, Homma KJ, Poo MM. 2004. Shrinkage of dendritic spines associated with long-term depression of hippocampal synapses. Neuron 44: $749-757$

Zito K, Knott G, Shepherd GM, Shenolikar S, Svoboda K. 2004. Induction of spine growth and synapse formation by regulation of the spine actin cytoskeleton. Neuron 44: 321-334.

Received February 3, 2010; accepted in revised form June 29, 2010. 


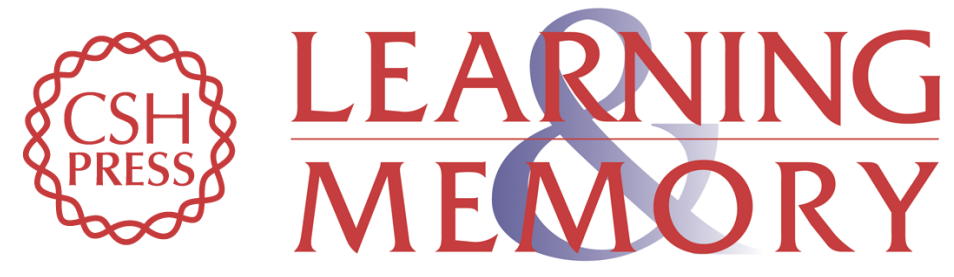

\section{Region-specific involvement of actin rearrangement-related synaptic structure alterations in conditioned taste aversion memory}

Ai-Ling Bi, Yue Wang, Bo-Qin Li, et al.

Learn. Mem. 2010, 17:

Access the most recent version at doi:10.1101//m.1772310

Supplemental http://learnmem.cshlp.org/content/suppl/2010/08/20/17.9.420.DC1
Material

References This article cites 45 articles, 19 of which can be accessed free at: http://learnmem.cshlp.org/content/17/9/420.full.html\#ref-list-1

License

Email Alerting Receive free email alerts when new articles cite this article - sign up in the box at the Service top right corner of the article or click here. 